\title{
Approximate State Transition Matrix and Secular Orbit Model
}

\author{
M. P. Ramachandran \\ Flight Dynamics Group, ISRO Satellite Centre, Bangalore 560 017, India \\ Correspondence should be addressed to M. P. Ramachandran; mprama@gmail.com
}

Received 21 September 2014; Revised 24 February 2015; Accepted 24 February 2015

Academic Editor: Christopher J. Damaren

Copyright (C) 2015 M. P. Ramachandran. This is an open access article distributed under the Creative Commons Attribution License, which permits unrestricted use, distribution, and reproduction in any medium, provided the original work is properly cited.

\begin{abstract}
The state transition matrix (STM) is a part of the onboard orbit determination system. It is used to control the satellite's orbital motion to a predefined reference orbit. Firstly in this paper a simple orbit model that captures the secular behavior of the orbital motion in the presence of all perturbation forces is derived. Next, an approximate STM to match the secular effects in the orbit due to oblate earth effect and later in the presence of all perturbation forces is derived. Numerical experiments are provided for illustration.
\end{abstract}

\section{Introduction}

Autonomous orbit control in satellites is possible with the present onboard technological advancements. The Global Positioning System receiver solution gives the satellite position measurement in Cartesian frame. State propagating equations along with the measurement equations in the linear filter then estimate the orbit. State transition matrix (STM) is used in the state update equations. A reference orbit model is available onboard. Using the receiver orbit solution the absolute orbit control system then ensures the satellite motion to this reference orbit model in the earth centered fixed Cartesian reference frame. This control enables the satellite to achieve the required orientation too. In orbit determination system, STM of two-body dynamics as suggested in [1] is usually used. Yet it will be always desirable to match the complete dynamics especially to improve the accuracy and scalability of the navigation system [2].

The orbital motion of the satellite is made up of secular or mean motion along with short and long periodic motions [3, page 571]. When we include the complete dynamics as reference orbit we have to use continuous control. This requires more fuel. Continuous maneuver can also disturb the payload functioning. On the other hand mean motion (without periodic motions) as a reference orbit is more suited for orbit control by impulse thrusting. This is adopted in formation flying [4, Chap 10] besides that the mean motion is used to derive the initial conditions. It is noted that orbit control is usually executed as a function of time [5] instead of true anomaly. In the control system, the state measurements in Cartesian frame are usually updated in time space. Subsequently the STM derived here then updates these states.

STM henceforth shall mean absolute STM unless mentioned. It may be noted that Vallado [[3] page 748] has discussed the STM for two-body orbital motion. In [6] a STM including the oblate earth effects using equinoctial mean elements and then applying interpolation is obtained. The present note brings out a STM that is in Cartesian frame as an alternative to [6] and considers only secular effects. We note in the literature that the STM that is in Cartesian frame is derived in [7] and it includes secular and periodic effects. Here the periodic effects are neglected. Further the STM derived here is extendable to accommodate secular along track effects in the presence of all perturbations. This is simpler than the expansion based method of deriving the STM as in [8].

It is important to note that secular forces due to oblate effect are considered in relative motion as in formation flying which is based on geometric approach [9]. The absolute transition matrix derived here can further be used to derive relative transition matrix as in [10]. This work is beyond the scope of this paper. 


\section{Secular Acceleration}

Consider the equation of motion

$$
\ddot{\mathbf{r}}=\nabla U+\mathbf{a}_{\mathbf{p}}
$$

where $\ddot{\mathbf{r}}$ denotes the second derivative with respect to time of $\mathbf{r}=(x, y, z)$, the position vector in the inertial frame. The disturbing potential [11] is

$$
U(r, \psi, z)=\left(\frac{\mu}{r}\right)-\left(\frac{\mu}{r}\right)\left(\frac{R}{r}\right)^{2} J_{2}\left(\frac{1}{2}\right)\left\{3 \sin ^{2} \delta-1\right\},
$$

where $J_{2}=0.0010826, \delta$ is the instantaneous declination, $R$ is the radius of the earth, $\mu$ is the gravitational constant, and $r$ is the magnitude of the position vector, $\mathbf{r}$. The vector $\mathbf{a}_{\mathbf{p}}=$ $\left(a_{p x}, a_{p y}, a_{p z}\right)$ represents other perturbation forces due to the inhomogeneous mass distribution of the earth, third body forces due to sun and moon besides solar radiation pressure and atmosphere drag forces. The potential is axisymmetric about the $z$-axis and is independent of azimuth angle $\psi$. The Lagrange's planetary equation of motion is invoked and the following relations are deduced. The Keplerian elements are averaged over an orbit. The first-order secular motion that neglects periodic effects is described by

$$
\begin{aligned}
& a=a_{0}, \\
& e=e_{0}, \\
& i=i_{0},
\end{aligned}
$$

where $a, e, i$ are, respectively, the semimajor axis, eccentricity, and inclination are invariant over the duration of interest:

$$
\begin{gathered}
w=w_{0}+\left(\frac{3}{2}\right) J_{2}\left(\frac{R}{p}\right)^{2} \check{n}\left\{2-\left(\frac{5}{2}\right) \sin ^{2}(i)\right\} \Delta t, \\
\Omega=\Omega_{0}-\left(\frac{3}{2}\right) J_{2}\left(\frac{R}{p}\right)^{2} \check{n} \cos (i) \Delta t, \\
\bar{n}=n_{0}\left[1+\left(\frac{3}{2}\right) J_{2}\left(\frac{R}{p}\right)^{2}\left[1-\left(\frac{3}{2}\right) \sin ^{2}(i)\right)\left(1-e^{2}\right)^{1 / 2}\right], \\
n_{0}=\sqrt{\left(\frac{\mu}{a^{3}}\right)}, \\
M=M_{0}+\check{n} t .
\end{gathered}
$$

And $w, \Omega, M$ are argument of perigee, the longitude of ascending node, and mean anomaly, respectively. The equation of the centre enables getting the true anomaly $(f)$ :

$$
\begin{aligned}
f= & M+\left(2 e-\left(\frac{1}{4}\right) e^{3}\right) \sin (M)+\left(\frac{5}{4}\right) e^{2} \sin (2 M) \\
& +\left(\frac{13}{12}\right) e^{3} \sin (3 M)+o\left(e^{4}\right) .
\end{aligned}
$$

In (3a) and (3b) we note that

$$
p=a\left(1-e^{2}\right)
$$

Equation (4) is used when the eccentricity is not large. The longitude of the ascending node $\Omega_{0}$ varies linearly with incremental time, $\Delta t$. Orbit models in the satellite for control purposes need to have the cross-track motion that is predominant due to $J_{2}$. The argument of perigee $(w)$ along with the true anomaly $(f)$ gives the argument of latitude $(\theta)$ which is

$$
\theta_{2}=w_{2}+f_{2}
$$

Here, the subscript 2 has been added to denote the $J_{2}$ model. When all forces are included and solution of (1) is obtained, the instantaneous argument of latitude is denoted by $\theta_{a}$. Here, in this paper, a proposal is made to add a polynomial function to the argument of latitude $\theta_{2}$, in (6) over every orbit. This is a mean variation of the differential argument of latitude and could be, say, a quadratic or cubic power of time and is denoted as $\theta_{f}$ [[3] page 570,652$]$. Here, in this note, $\theta_{f}$ is a least squares fit over one orbital period and it accommodates the secular difference. This is defined as

$$
\theta_{p} \stackrel{\text { def }}{=} \theta_{2}+\theta_{f} \text {. }
$$

The residue between $\theta_{a}$ and $\theta_{p}$ is periodic, which is incidentally not required for control. This correction enables (3a) and (3b) along with (7) to match the secular effects when all perturbation is present to a reasonable accuracy specially along track. Next a STM that matches the orbit model in (3a) and (3b) and later the secular effect in the presence of all forces is derived.

The following equations are used to transform from the orbital frame $[r, t, n]$ to the Cartesian frame $[x, y, z]$ :

$$
\begin{gathered}
\left(\begin{array}{c}
\bar{x} \\
\bar{y} \\
\check{z}
\end{array}\right)=(A)\left(\begin{array}{c}
\check{r} \\
\check{t} \\
\check{n}
\end{array}\right) \\
a_{1,1}=\cos (\theta) \cos (\Omega)-\cos (i) \sin (\Omega) \sin (\theta) \\
a_{1,2}=-\sin (\theta) \cos (\Omega)-\cos (i) \sin (\Omega) \cos (\theta) \\
a_{1,3}=\sin (i) \sin (\theta) \\
a_{2,1}=\cos (\theta) \sin (\Omega)+\cos (i) \cos (\Omega) \sin (\theta) \\
a_{2,2}=-\sin (\theta) \sin (\Omega)+\cos (i) \cos (\Omega) \cos (\theta) \\
a_{2,3}=-\sin (i) \cos (\Omega) \\
a_{3,1}=\sin (\theta) \sin (i) \\
a_{3,2}=\sin (i) \cos (\theta) \\
a_{3,3}=\cos (i),
\end{gathered}
$$

where unit vectors $\check{r}, \check{t}, \check{n}$ respectively are in the radial, tangential, that is along the direction of motion (along-track) and normal to the orbital plane (see Figure 1).

By using $\sin (\delta)=r \sin (\theta) \sin (i)$ in $(2)$, the potential due to oblate earth effect becomes

$$
U_{2}=-\left(\frac{\mu}{r}\right)\left(\frac{R}{r}\right)^{2} J_{2}\left(\frac{1}{2}\right)\left\{3 \sin ^{2}(i) \sin ^{2}(\theta)-1\right\} .
$$




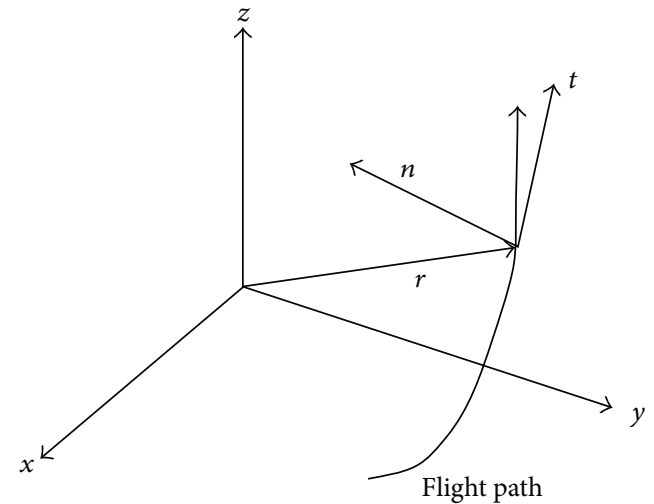

FIGURE 1: Orbit frame from position, tangential, and normal vectors.

The acceleration is then obtained using the relations in (8) and is

$$
\begin{aligned}
\nabla U= & \left(-\frac{\mu}{r^{2}}\right)(\widetilde{r})-\left(\mu R^{2} J_{2}\left(\frac{1}{2}\right)\right)\left\{\left(-\frac{15 z^{2}}{r^{6}}\right)+\left(\frac{3}{r^{4}}\right)\right\}(\widetilde{r}) \\
& -\left(\mu R^{2} J_{2}\left(\frac{1}{2}\right)\right)\left\{\frac{6 z}{r^{5}}\right\}(\widetilde{z}) .
\end{aligned}
$$

Equation (11) when substituted into (8) finally gives the accelerations in the $(x, y, z)$ frame:

$$
\begin{aligned}
& a_{x}=-\frac{\mu x}{\left(r^{3}\right)\left[1+(3 / 2) J_{2}(R / r)^{2}\left(1-\left(5 z^{2} / r^{2}\right)\right)\right]}, \\
& a_{y}=\left(\frac{y}{x}\right) a_{x}, \\
& a_{z}=-\frac{\mu z}{\left(r^{3}\right)\left[1+(3 / 2) J_{2}(R / r)^{2}\left(3-\left(5 z^{2} / r^{2}\right)\right)\right]} .
\end{aligned}
$$

Partial differentiation with respect to the state variables $(x, y, z)$ in (12) yields the STM that includes both secular and periodic components (see [7]).

Here, the term $\cos (2 \theta)$ in (10) is periodic and neglected; the remaining term that is secular is retained. In (2) we have

$$
U(r, \delta)=\left(\frac{\mu}{r}\right)-\left(\frac{\mu}{r}\right)\left(\frac{R}{r}\right)^{2} J_{2}\left(\frac{1}{2}\right)\left\{\left(\frac{3}{2}\right) \sin ^{2}(i)-1\right\} .
$$

Note that the potential in (13) is independent of $(z)$. The net acceleration then is obtained as in (11) by denoting $\alpha=$ $-\mu R^{2} J_{2}(3 / 2)\left(1-(3 / 2) \sin ^{2}(i)\right)$ in Cartesian frame and is given by

$$
\begin{aligned}
& a_{x}=\left(\frac{\alpha x}{r^{5}}\right)-\frac{\mu x}{r^{3}}, \\
& a_{y}=\left(\frac{\alpha y}{r^{5}}\right)-\frac{\mu y}{r^{3}}, \\
& a_{z}=\left(\frac{\alpha z}{r^{5}}\right)-\frac{\mu z}{r^{3}} .
\end{aligned}
$$

Then

$$
\begin{gathered}
\frac{\partial a_{x}}{\partial x}=\left(-\frac{3 \alpha}{r^{5}}\right)+\left(\frac{15 \alpha x^{2}}{r^{7}}\right)+\frac{3 \mu x^{2}}{r^{5}}-\frac{\mu}{r^{3}} \\
\frac{\partial a_{x}}{\partial y}=\left(\frac{15 \alpha x y}{r^{7}}\right)+\frac{3 \mu x y}{r^{5}}, \\
\frac{\partial a_{x}}{\partial z}=\left(\frac{15 \alpha x z}{r^{7}}\right)+\frac{3 \mu x z}{r^{5}}, \\
\frac{\partial a_{y}}{\partial y}=\frac{\partial a_{x}}{\partial y}, \\
\left(-\frac{3 \alpha}{r^{5}}\right)+\left(\frac{15 \alpha x^{2}}{r^{7}}\right)+\frac{3 \mu y^{2}}{r^{5}}-\frac{\mu}{r^{3}} \\
\frac{\partial a_{y}}{\partial z}=\left(\frac{15 \alpha y z}{r^{7}}\right)+\frac{3 \mu y z}{r^{5}}, \\
\frac{\partial a_{z}}{\partial x}=\frac{\partial a_{x}}{\partial z}, \\
\frac{\partial a_{z}}{\partial y}=\frac{\partial a_{y}}{\partial z}, \\
\left(-\frac{15 \alpha z^{2}}{r^{5}}\right)+\frac{3 \mu z^{2}}{r^{5}}-\frac{\mu}{r^{3}}
\end{gathered}
$$

It may be noted that when $\theta$ is substituted into (8) by $\theta_{2}$ in (3a) and (3b) or $\theta_{p}$ in (7), the accelerations derived in (15) are still valid. This implies that the STM (to be derived in the section) with the accelerations derivatives in (15) are valid for (1) without considering periodic effects.

\section{Approximate STM}

Next use the total acceleration in (14) and derive the approximate STM following Markley [12]. The STM is then obtained approximately based on Taylor expansion

$$
\Phi\left(t, t_{0}\right)=\left(\begin{array}{ll}
\Phi_{r r} & \varphi_{r v} \\
\Phi_{v r} & \varphi_{v v}
\end{array}\right)
$$

With a knowledge of initial states as in (3a) and (3b) at $t_{0}$, the matrix $\Phi$ can be used to obtain state at the subsequent instant " $t$," using
$X(t)=\Phi\left(t, t_{0}\right) X\left(t_{0}\right)$ 


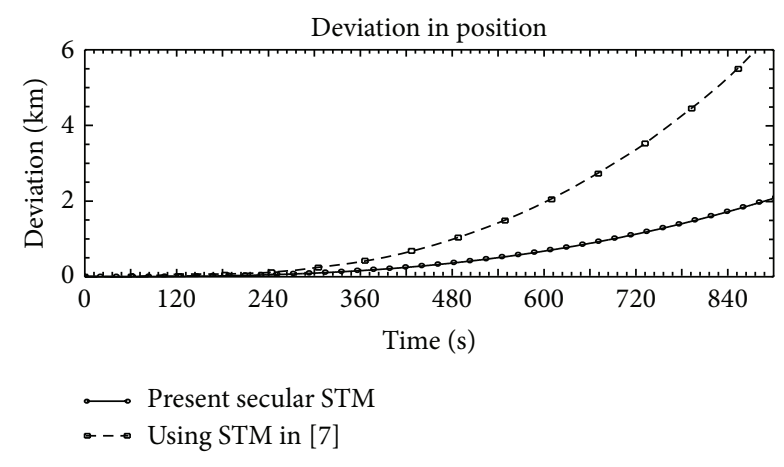

FIGURE 2: Deviation in position for Molniya orbit.

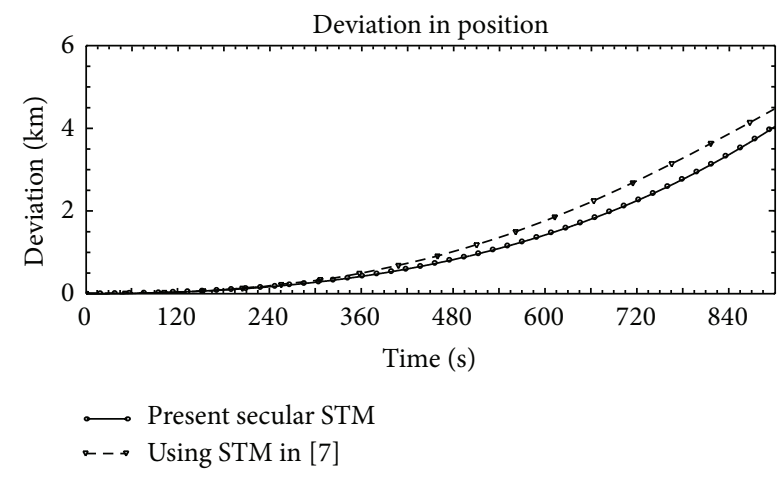

FIgURE 3: Deviation in position for sun-synchronous orbit.

where $X(t)$ is differential of the states $\left(x, y, z, x^{\prime}, y^{\prime}, z^{\prime}\right)$ at $t_{0}$. Discarding higher order terms in (16) we have

$$
\begin{gathered}
\Phi_{r r}=I+\frac{\left(2 \mathbf{G}_{0}+\mathbf{G}\right)(\Delta t)^{2}}{6}, \\
\Phi_{r v}=I \Delta t+\frac{\left(2 \mathbf{G}_{0}+\mathbf{G}\right)(\Delta t)^{3}}{12}, \\
\Phi_{v r}=\frac{\left(\mathbf{G}_{0}+\mathbf{G}\right)(\Delta t)}{2}, \\
\Phi_{r v}=I+\frac{\left(\mathbf{G}_{0}+2 \mathbf{G}\right)(\Delta t)^{2}}{6} .
\end{gathered}
$$

The gradient matrix is

$$
\mathbf{G}=\left(\begin{array}{lll}
\frac{\partial a_{x}}{\partial x} & \frac{\partial a_{x}}{\partial y} & \frac{\partial a_{x}}{\partial z} \\
\frac{\partial a_{y}}{\partial x} & \frac{\partial a_{y}}{\partial y} & \frac{\partial a_{y}}{\partial z} \\
\frac{\partial a_{z}}{\partial x} & \frac{\partial a_{z}}{\partial y} & \frac{\partial a_{x}}{\partial z}
\end{array}\right) .
$$

The matrices $\mathbf{G}_{0}$ and $\mathbf{G}$ denote $\mathbf{G}\left(t_{0}\right)$ and $\mathbf{G}(t)$, respectively, and $\Delta t$ is $\left(t-t_{0}\right)$.

\section{Illustration}

STM is not to be used as a propagator and is used between the updates of the state over the duration of orbit determination.
However, an experiment of propagation is carried out here over certain duration to ensure that the present model efficiently captures the secular effects. The selected orbit is Molniya orbit with $a=26554 \mathrm{kms}, e=0.72$, and $i=63.4$ degrees. This orbit is more eccentric and the short periodic effects are predominant. The top dotted line in Figure 2 is the result that depicts the absolute deviation in position (distance) from the STM that includes short and periodic effects given in [7] with respect to (3a) and (3b). The bottom curve is the absolute deviation between the proposed STM (15)-(19) with respect to (3a) and (3b) again as function of time. Both have the same initial conditions. This illustrates that the proposed model is closer to the mean or secular orbital motion given in (3a) and (3b) in the presence of $J_{2}$ effect neglecting the numerical error due to propagation. The numerical propagation error due to step size is common in both. This validates that the STM derived in Section 3 using the partial derivatives of accelerations in (15) contains secular effects alone.

Next a similar exercise as carried out in Figure 2 is considering a sun-synchronous polar orbit with $a=7168.4$, $e=0.0011$, and $i=98.51$. The top dashed line in Figure 3 is the result that depicts the deviation in position from the STM that includes short and periodic effects. The bottom curve is the deviation in position from the proposed STM. The illustration confirms that the proposed model is closer to (3a) and (3b).

Next example is about illustrating the modeling of (7). The satellite orbit has $a=7244.3, e=0.0003$, and $i=20$ degrees. The satellites placed in such orbits are mostly used for metrological purposes. The payload sensor usually covers a wide area as in microwave remote sensing applications. The argument of latitude $\theta_{2}$ corresponding to ( $3 a$ ) and (3b) is first derived. The satellite motion is also obtained as solution of (3a) and (3b) while considering complete perturbations as mentioned in (1). The instantaneous argument of latitude is $\theta_{a}$. From (3a) and (3b) the argument of latitude, denoted as $\theta_{2}$, is obtained. The residue $\left(\theta_{a}-\theta_{2}\right)$ is plotted against time in Figure 4. It can be seen that this deviation is secular and has large effect along the track.

The difference between the argument of latitude of the full force model $\theta_{a}$ and $\theta_{2}$ over one orbit, that is, 102 minutes in duration, is then fit for the secular effect by a polynomial in a least square sense. For in this case it is

$$
\theta_{f}=\theta_{b}-0.03624+0.0012 t-0.000013 t^{2}+0.000000046 t^{3} .
$$

The same fit is proposed here to extend over successive orbits. However, at the end of each orbital period the ordinate value of difference $\theta_{b}$ is used for the next orbital period. Here, for example, the value of $\theta_{b}$ is -0.113 at the start. This approach of orbit model representation can be considered as an alternative to the existing methods for onboard applications [12].

The difference between the argument latitude of the full force model $\theta_{a}$ and $\theta_{f}$ in (7) is computed. This residue is illustrated in Figure 5 over four orbits and is observed to be periodic over each orbital period. The initial states for 


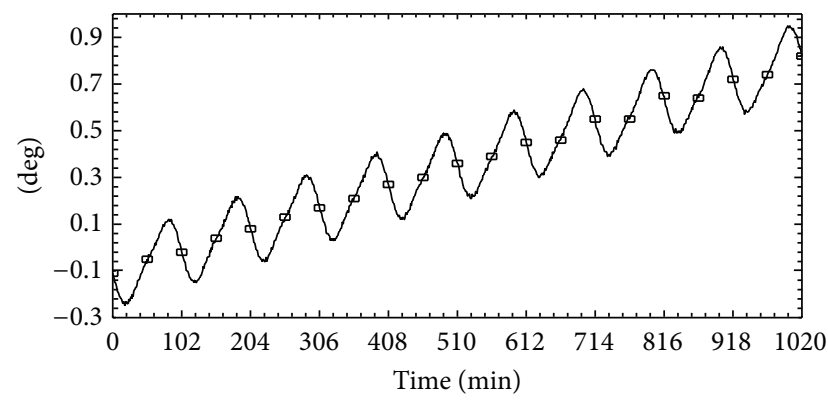

FIGURE 4: Difference in argument of latitude.

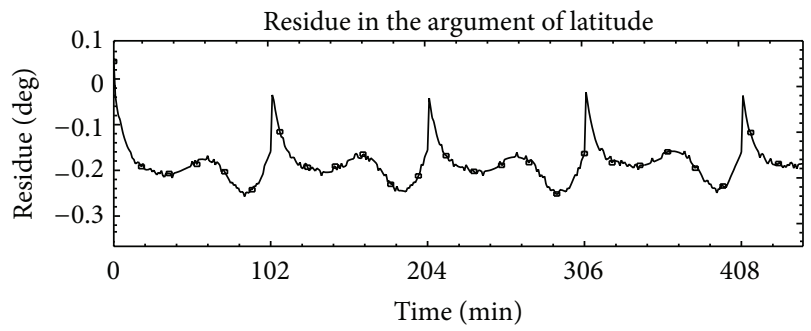

Figure 5: Residue with respect to the polynomial fit.

the STM are obtained from the proposed orbit model while using (3a) and (3b) along with the correction in (20). The absolute deviation is with respect to the position of full force secular propagation model. The plot in Figure 6 is similar to that in Figure 1 or Figure 2. The deviation in position in the plot uses the STM in Section 3 and using (15) can be seen to accommodate secular effects in the presence all perturbations.

\section{Application}

Equations (3a) and (3b) describe the dynamics of the orbiting satellite when oblate earth effect is considered. The relative motion based on geometric approach is given in [9]. They independently make use of the dynamics in (3a) and (3b) for both satellites. The two satellites have identical $(a, e, i)$ in (3a) and distinct $(w, \Omega, M)$ in (3b). The relative motion as reproduced from [9] is

$$
\begin{aligned}
\Delta x(\Delta t)= & -1+c^{2}\left(\frac{i_{m}}{2}\right) c^{2}\left(\frac{i_{s}}{2}\right) c(\delta \theta+\delta \Omega) \\
& +s^{2}\left(\frac{i_{m}}{2}\right) s^{2}\left(\frac{i_{s}}{2}\right) c(\delta \theta-\delta \Omega) \\
& +s^{2}\left(\frac{i_{m}}{2}\right) c^{2}\left(\frac{i_{s}}{2}\right) c\left(2 \theta_{m}+\delta \theta+\delta \Omega\right) \\
& +c^{2}\left(\frac{i_{m}}{2}\right) s^{2}\left(\frac{i_{s}}{2}\right) c\left(2 \theta_{1}+\delta \theta-\delta \Omega\right) \\
& +\frac{1}{2 s\left(i_{m}\right) s\left(i_{s}\right)\left[c(\delta \theta)-c\left(2 \theta_{m}+\delta \theta\right)\right]},
\end{aligned}
$$

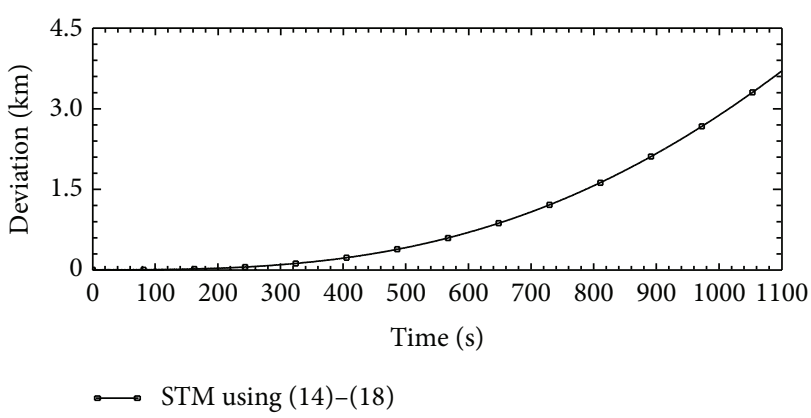

FIgURE 6: Deviation in position.

$$
\begin{aligned}
\Delta y(\Delta t)= & c^{2}\left(\frac{i_{m}}{2}\right) c^{2}\left(\frac{i_{s}}{2}\right) s(\delta \theta+\delta \Omega) \\
& +s^{2}\left(\frac{i_{m}}{2}\right) s^{2}\left(\frac{i_{s}}{2}\right) s(\delta \theta-\delta \Omega) \\
& -s^{2}\left(\frac{i_{m}}{2}\right) c^{2}\left(\frac{i_{s}}{2}\right) s\left(2 \theta_{m}+\delta \theta+\delta \Omega\right) \\
& +c^{2}\left(\frac{i_{m}}{2}\right) s^{2}\left(\frac{i_{s}}{2}\right) s\left(2 \theta_{m}+\delta \theta-\delta \Omega\right) \\
& +\frac{1}{2 s\left(i_{m}\right) s\left(i_{s}\right)\left[s(\delta \theta)+s\left(2 \theta_{m}+\delta \theta\right)\right]} \\
\Delta z(\Delta t)= & -s\left(i_{m}\right) s(\delta \Omega) c\left(\theta_{s}\right) \\
& -\left[s\left(\frac{i_{m}}{2}\right) c\left(i_{s}\right) c(\delta \Omega)-c\left(i_{m}\right) s\left(i_{s}\right)\right] s\left(\theta_{s}\right) .
\end{aligned}
$$

Here $\theta_{m}$ is the instantaneous argument of latitude, that is, $\theta_{2}$ of the main or chief satellite. Similarly $\theta_{s}$ is the instantaneous argument of latitude of the second or follower satellite and their difference $\left(\theta_{m}-\theta_{s}\right)$ which is denoted as $\delta \theta$. The differences in the instantaneous longitude of the ascending node between them is $\delta \Omega$. The $c$ and $s$ functions denote cos and sin functions and $i_{m}$ and $i_{s}$ denote the inclinations of the main and follower, respectively. Also $\Delta t$ denotes the incremental time as used in (3a) and (3b). In (21), $\Delta x, \Delta y, \Delta z$ are relative positions of the second satellite in the orbit frame of the first.

Here we shall outline the application of the secular approximation $\theta_{p}$ from (7) when substituted into (21). This is to enhance (21) to match the secular effects particularly along the track while considering all perturbations. To do so we replace the argument of latitude angle $\theta_{2}$ for both satellites in (21) by an appropriate $\theta_{p}$, computed individually using (20) and (7) as in Figure 6, for the main and secondary satellites. These computed $\theta_{p}$ are then substituted into (21) as $\overline{\theta_{m}}$ and $\widetilde{\theta}_{s}$, respectively, for the main and secondary satellite. Now, (21) is enhanced to match the secular effects particularly along the track of the full force model. It may be noted that in formation flying, (21) is used for guidance and can also be used to derive the initial conditions. 


\section{Conclusion}

Approximate orbit model that captures the secular motion in the argument of latitude while considering all perturbation forces is obtained. Approximate state transition matrix is first derived for the orbital motion that matches the secular motion with the effects of oblate earth. This STM can also accommodate the approximate orbit model with all forces. Numerical behavior of the state transition matrix and the orbit models has been provided. Finally a direct application of the orbit model in relative motion of two satellites has been indicated. The suggested approach is simpler for onboard implementation.

\section{Conflict of Interests}

The author declares that there is no conflict of interests regarding the publication of this paper.

\section{Acknowledgments}

The author acknowledges all the three anonymous referees for their comments that have enhanced the presentation. The author thanks Mr. N. S. Gopinath, Group Director, and Dr. S. K. Shivakumar, Director from ISRO Satellite Centre, for their encouragements.

\section{References}

[1] E. Gill, O. Montenbruck, and K. Brieß, "GPS-based autonomous navigation for the BIRD satellite," in Proceedings of the 15th International Symposium on Spaceflight Dynamics, Biarritz, France, June 2000

[2] S. D. Amico, J.-S. Ardaens, and O. Montenbruck, "Navigation of formation flying spacecraft using GPS: the PRISMA technology demonstration," in Proceedings of the 22nd International Technical Meeting of the Satellite Division of the Institute of Navigation (ION GNSS '09), pp. 1427-1441, Savannah, Ga, USA, September 2009.

[3] D. A. Vallado, Fundamentals of Astrodynamics and Applications, Kluwer Academic Publishers, Dordrecht, The Netherlands, 2001.

[4] K. T. Alfriend, S. R. Vadali, P. Gurfil, J. P. How, and L. S. Breger, Spacecraft Formation Flying, Elsevier, Oxford, UK, 2010.

[5] H. Schaub, S. R. Vadali, J. L. Junkins, and K. T. Alfriend, "Spacecraft formation flying control using mean orbit elements," The Journal of the Astronautical Sciences, vol. 48, no. 1, pp. 69-87, 2001.

[6] J. S. Shaver, Formulation and evaluation of parallel algorithms for orbit determination problem [Ph.D. thesis], Department of Aeronautics, Massachusetts Institute of Technology, Cambridge, Mass, USA, 1980.

[7] A. P. M. Chiaradia, H. K. K. Kuga, and A. F. B. A. Prado, "Comparison between two methods to calculate the transition matrix of orbit motion," Mathematical Problems in Engineering, vol. 2012, Article ID 768973, 12 pages, 2012.

[8] Y. Tsuda, "State transition matrix approximation with geometry preservation for general perturbed orbits," Acta Astronautica, vol. 68, no. 7-8, pp. 1051-1061, 2011.
[9] S. R. Vadali, "An analytical solution of relative motion of satellites," in Proceedings of the Dynamics and Control of Systems and Structures in Space Conference, Cranfield, UK, July 2002.

[10] C. J. Damaren, "Almost periodic relative orbits under $\mathrm{J}_{2}$ perturbations," Proceedings of the Institution of Mechanical Engineers, Part G: Journal of Aerospace Engineering, vol. 221, no. 5, pp. 767774, 2007.

[11] A. E. Roy, Orbital Motion, Adam Hilger, Bristol, UK, 1982.

[12] F. L. Markley, "Approximate Cartesian state transition matrix," The Journal of Astronautical Sciences, vol. 34, no. 2, pp. 161-169, 1986. 

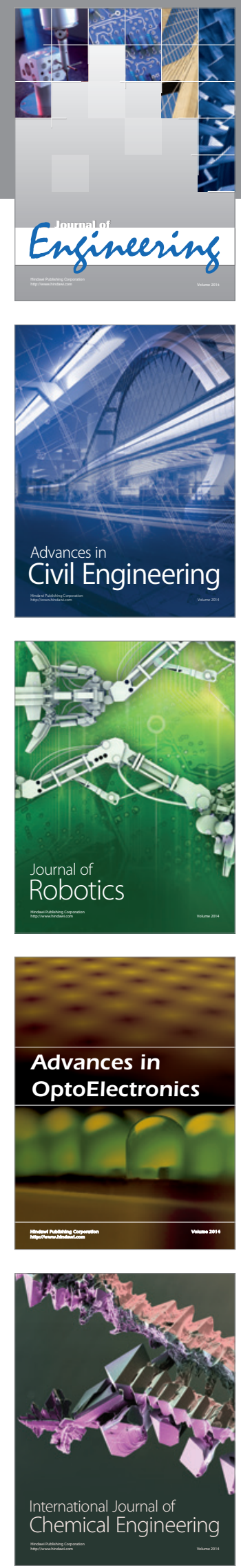

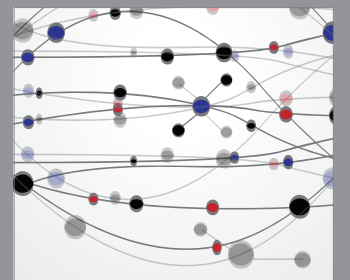

The Scientific World Journal
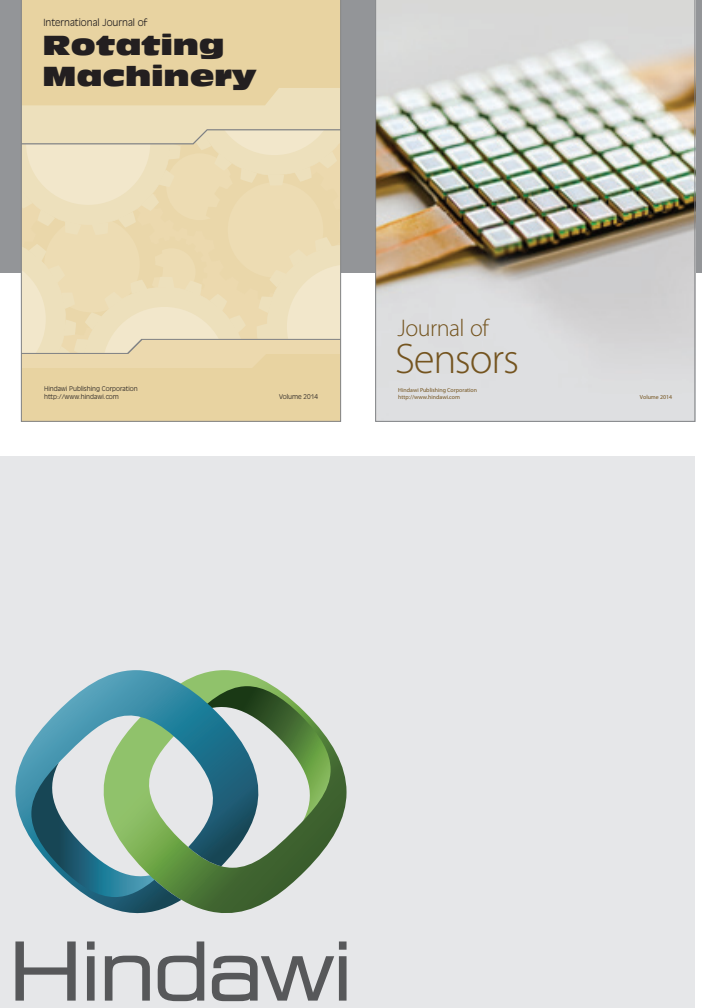

Submit your manuscripts at http://www.hindawi.com
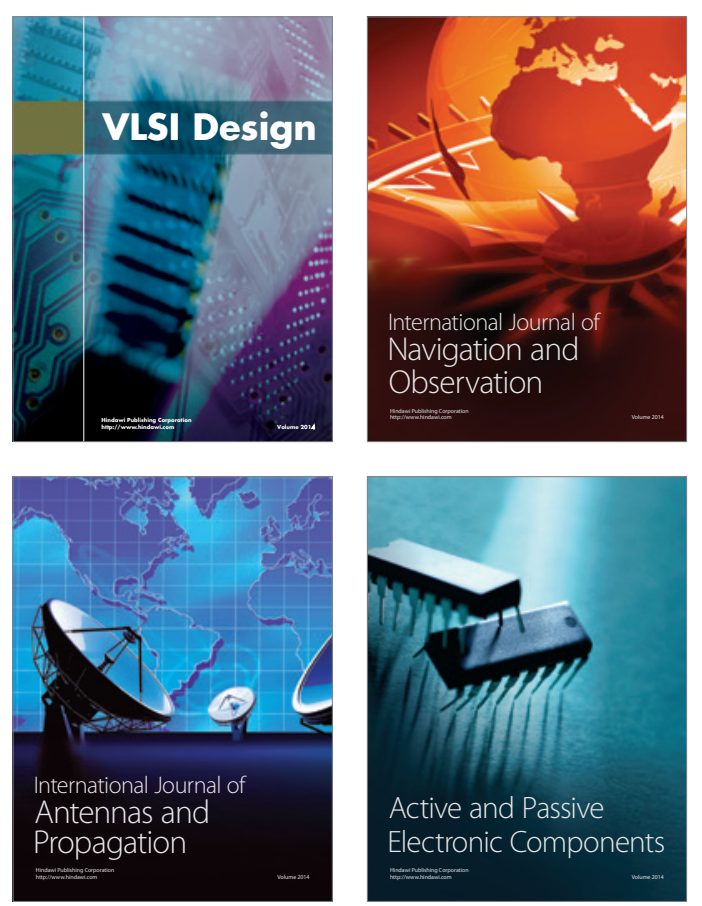
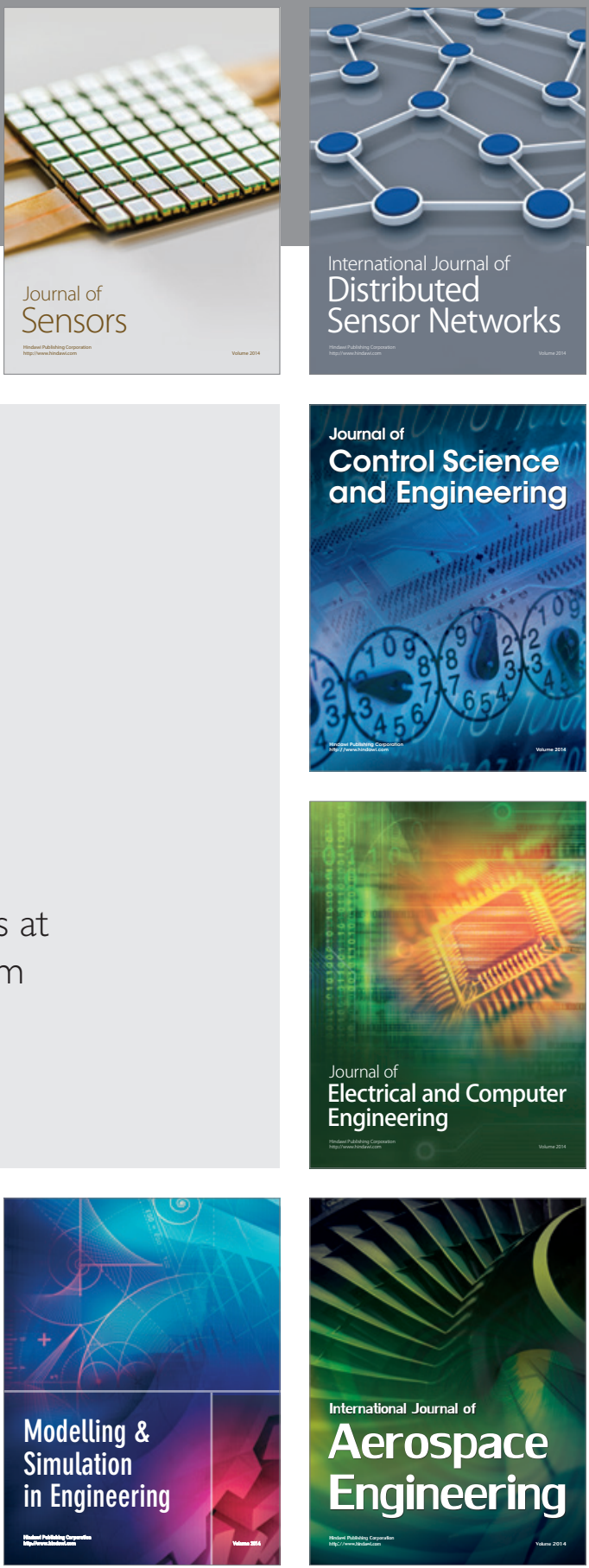

Journal of

Control Science

and Engineering
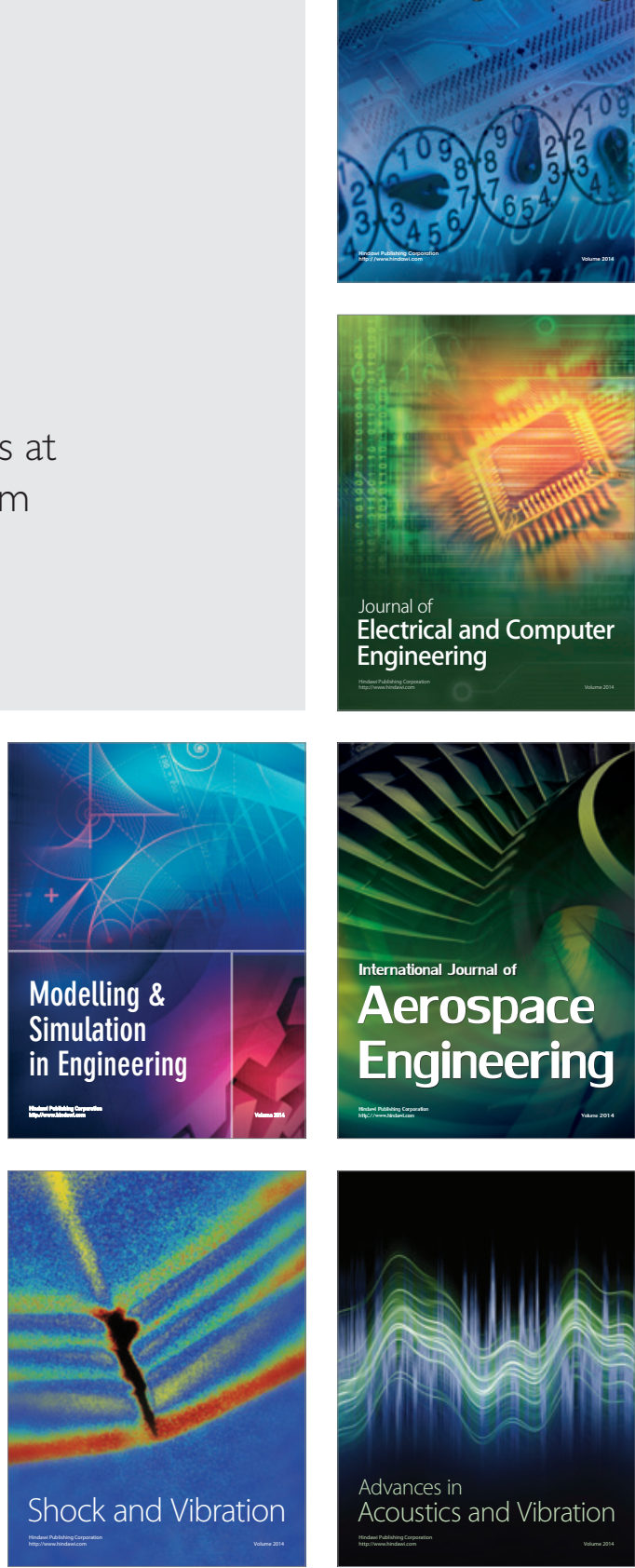